\title{
Vergleichende Evaluation zur Usability mobiler Web-Browser
}

\author{
Lukas Lamm, Manuel Burghardt, Christian Wolff \\ Lehrstuhl für Medieninformatik, Universität Regensburg
}

\section{Zusammenfassung}

Dieser Beitrag beschreibt eine vergleichende Usability-Evaluation der drei meistgenutzten Web-Browser für mobile Endgeräte. Dabei werden die zuvor identifizierten Hauptfunktionen der Browser durch entsprechend konstruierte Aufgaben mit sechs Studienteilnehmern ausführlich getestet. Die Ergebnisse werden in Form von think aloud-Protokollen sowie einer Abwandlung des User Experience Questionnaire (UEQ) dokumentiert und abschließend diskutiert.

\section{Mobile Usability}

Der in den letzten Jahren deutlich erkennbare Anstieg von internetfähigen Mobiltelefonen auf dem Markt bringt auch neue Anforderungen an die Benutzerfreundlichkeit von Webseiten auf mobilen Endgeräten mit sich. Dabei existieren zahlreiche Studien, welche die Relevanz mobiler Web-Browser als Zugangsmethode zum Internet belegen. Während einer Studie der Firma Accenture zufolge im Jahr 2011 bereits $28 \%$ der Teilnehmer angaben, ihr Smartphone für den mobilen Internetzugang zu nutzen (Accenture, 2011), waren es im Folgejahr bereits $50 \%$ der Befragten (Accenture, 2012). Eine weitere Studie der Unternehmen Google und Nielsen zeigt, dass 77 \% der Suchanfragen auf mobilen Geräten in Situationen erfolgen, in denen gleichzeitig auch ein Desktop-PC zur Verfügung steht (Google \& Nielsen, 2013). Dabei beeinflussen zwei wesentliche Faktoren die Usability von Webseiten auf mobilen Endgeräten: Einerseits müssen Inhalte, Navigations- und Interaktionskonzepte im Bereich des Webdesigns so angepasst werden, dass sie gut auf kleinen Bildschirmen dargestellt und ohne Eingabegeräte wie etwa Maus und Tastatur bedient werden können. Dazu haben sich in der Webdesign-Praxis Paradigmen wie etwa mobile first (Wroblewski, 2011) oder responsive design (Gardner 2011, Marcotte 2010) etabliert. Andererseits spielt als zweiter Usability-Faktor auch der Browser, in dem die mobile Webseite dargestellt wird, eine wichtige Rolle. 
Bislang gibt es nur wenige Usability-Studien zu mobilen Web-Browsern: So untersuchen Roto \& Kaikkonen (2003) den Web-Browser des Nokia 7650. Zum Zeitpunkt der Studie bestanden jedoch wesentliche Einschränkungen bezüglich der Hardware, wie etwa die geringe Bildschirmauflösung von $176 \times 208$ Pixeln und die Verfügbarkeit von nur 4096 Farben. In einer weiteren Studie wurde die Nutzungserfahrung von mobilen Browsern mit denen auf Desktop-PCs verglichen (Shrestha, 2007). Die Eingabemethode durch einen Joystick führte im Vergleich zur gewohnten Bedienung eines Browsers mit Tastatur und Maus zu UsabilityProblemen. Voigts, Christmann \& Hagenhoff (2011) führen verschiedene Ein- und Ausgabemethoden für die nutzerfreundliche Gestaltung von Web-Browsern auf; zudem werden mobile Web-Browser hinsichtlich technischer Aspekte der Seitendarstellung vorgestellt. Eine vergleichende Analyse der Interaktionsmöglichkeiten zeigt, dass sich die Usability zwischen den Endgeräten deutlich unterscheidet.

\section{Ziel und Vorgehen}

Ziel dieses Beitrags ist es, durch eine vergleichende Evaluation der Hauptfunktionen mobiler Web-Browser in Hinblick auf deren Usability wesentliche Aspekte benutzerfreundlicher Browser herauszuarbeiten, um so einerseits Entwicklern Anhaltspunkte für künftige Weiterentwicklungen zu geben, und andererseits Anwendern die Auswahl eines geeigneten mobilen Browsers zu erleichtern. In unserer Studie werden die drei am häufigsten verwendeten Smartphone-Browser mit Methoden des Usability Testing untersucht. Zur deren Ermittlung wurden unterschiedliche Statistik-Portale mit Nutzungsdaten zu mobilen Browsern ausgewertet (Kap. 3.1). Anschließend wurden die wesentlichen Funktionen mobiler Web-Browser mittels einer Explorationsstudie ermittelt (Kap. 3.2). Die Ergebnisse der Funktionsanalyse dienen der Formulierung von Tasks für die anschließenden Usability-Evaluation (Kap. 3.3 3.6). In der Evaluationsstudie wurde die Umsetzung der Funktionen in den einzelnen Browsern vergleichend mit sechs Nutzern analysiert. Dazu wurden neben qualitativen Daten in Form von Screenvideos und think aloud-Protokollen auch quantitative Daten mit einer für die Evaluation angepassten Version des User Experience Questionnaires (UEQ, vgl. Laugwitz et al. 2006) erhoben. Die Ergebnisse der Erhebung werden in Kap. 4 näher diskutiert; Kap. 5 fasst die Studie in einem kurzen Fazit zusammen.

\section{Evaluationsdesign}

Um die Usability mobiler Browser zu erfassen, wurde ein Experimentaldesign entwickelt, das im folgenden Abschnitt vorgestellt wird. Zunächst wird beschrieben, welche Browser als Testobjekte ausgewählt wurden. Im Anschluss daran werden wesentliche Funktionen mobiler Browser diskutiert, die als Grundlage für die Evaluations-Tasks dienen. Neben der Wahl der Testpersonen und dem Versuchsaufbau werden schließlich der Ablauf und die verwendeten Testmaterialien erläutert. 


\subsection{Browser für mobile Endgeräte}

Die Internetseite StatCounter ${ }^{l}$ bietet neben weiteren Statistiken zu verwandten Themen auch eine Übersicht über die am häufigsten verwendeten mobilen Web-Browser. Der Anbieter misst absolute Zugriffswerte auf über drei Millionen Partnerwebseiten. Eine weitere Datenquelle zur Browsernutzung ist die Webseite NetMarketShare ${ }^{2}$. Wie auch bei StatCounter werden die Nutzungsdaten über Partnerwebseiten ermittelt, allerdings liegen bei NetMarketShare mit 40.000 Webseiten weniger Daten zur Auswertung zugrunde. Zudem misst NetMarketShare nicht absolute Zugriffszahlen, sondern Aufrufe pro Person. Tabelle 1 liefert einen Überblick über die drei am häufigsten genutzten, mobilen Web-Browser laut der beiden beschriebenen Statistikportale. Eine Analyse der wichtigsten Merkmale und Funktionen mobiler Browser folgt im nächsten Abschnitt.

\begin{tabular}{|l|l|l|l|l|}
\hline Browser & Hersteller & Veröffentlichung & Engine & $\begin{array}{l}\text { Betriebssystem- } \\
\text { Kompatabilität }\end{array}$ \\
\hline Safari & Apple Inc. & Juni 2007 & WebKit & iOS \\
\hline $\begin{array}{l}\text { Android Stock } \\
\text { Browser }\end{array}$ & Google Inc. & September 2008 & WebKit & Android \\
\hline Opera Mini & $\begin{array}{l}\text { Opera Software } \\
\text { ASA }\end{array}$ & Herbst 2005 & $\begin{array}{l}\text { Small-Screen-Rendering } \\
\text { über Proxy-Server }\end{array}$ & $\begin{array}{l}\text { iOS, Android, Java- } \\
\text { fähige Smartphones }\end{array}$ \\
\hline
\end{tabular}

Tabelle 1: Übersicht zu den drei am häufigsten genutzten, mobilen Web-Browsern.

\subsection{Merkmale und Funktionen mobiler Browser}

Um die Funktionen moderner Browser für das Experimentaldesign systematisch zu erfassen, wurde eine Exploration der drei meistverwendeten Browser durchgeführt. Dabei erfolgte die Erfassung in Form eines explorativen Walkthroughs der einzelnen Browser (Tabelle 2).

\begin{tabular}{|c|c|c|c|}
\hline $\begin{array}{c}\text { Generische Funk- } \\
\text { tionsbeschreibung }\end{array}$ & Apple Safari & Android Stock Browser & Opera Mini \\
\hline URL-Eingabe & Adressleiste & Omnibox & Adressleiste \\
\cline { 2 - 2 } Suchmaschinen-Suche & Suchleiste & Such- oder Adressleiste \\
\hline $\begin{array}{c}\text { Vorwärts-/Rückwärts- } \\
\text { Navigation }\end{array}$ & $\begin{array}{c}\text { Buttons in unterer } \\
\text { Leiste }\end{array}$ & $\begin{array}{c}\text { Im Optionsmenü, Android } \\
\text { Zurück-Taste }\end{array}$ & Buttons in unterer Leiste \\
\hline Tabs/Fenster & $\begin{array}{c}\text { Button in unterer } \\
\text { Leiste }\end{array}$ & Button neben Suchleiste & Button in unterer Leiste \\
\hline Lesezeichen & Lesezeichenmanager & Im Optionsmenü & $\begin{array}{c}\text { Button in der } \\
\text { Adressleiste, } \\
\text { Lesezeichenmanager }\end{array}$ \\
\hline
\end{tabular}

${ }^{1}$ StatCounter: Top 9 Mobile Browsers from Jan 2009 to Aug 2013, http://gs.statcounter.com/\#mobile_browser-wwmonthly-200901-201308, letzter Zugriff am 18.6.2014

2 NetMarketShare: Mobile browser market share, http://www.netmarketshare.com (Timeframe: 2008 - 2013), letzter Zugriff am 19.3.2014 


\begin{tabular}{|c|c|c|c|}
\hline Verlauf & $\begin{array}{c}\text { Im Lesezeichen- } \\
\text { Menü }\end{array}$ & Im Lesezeichenmanager & Im Optionsmenü \\
\hline Seite durchsuchen & Über Suchleiste & Im Optionsmenü & Im Optionsmenü \\
\hline Teilen & $\begin{array}{c}\text { Teilen-Menü } \\
\text { (beschränkt) }\end{array}$ & $\begin{array}{c}\text { Im Optionsmenü (Apps } \\
\text { können Eintrag } \\
\text { hinzufügen) }\end{array}$ & $\begin{array}{c}\text { Im Optionsmenü } \\
\text { (beschränkt) }\end{array}$ \\
\hline Offline-Zugriff & Leseliste & Im Optionsmenü & Im Optionsmenü \\
\hline Desktop-Version & Nicht verfügbar & Im Optionsmenü & Nicht verfügbar \\
\hline
\end{tabular}

Tabelle 2: Hauptfunktionen der drei meistgenutzten, mobilen Browser.

\subsection{Testpersonen}

Um den Ablauf der Usability-Evaluation und die Verständlichkeit der Tasks zu überprüfen, wurde ein Pretest mit einer Testperson durchgeführt. Da sich keine Probleme bei der Durchführung ergaben, wurden die Daten des Pretests ebenfalls für die Auswertung verwendet. Für die Tests wurden weitere fünf Testpersonen herangezogen. Laut Nielsen (1998) ist diese Anzahl an Testpersonen ausreichend, um etwa $80 \%$ der vorhandenen Usability-Probleme aufzudecken (guerilla usability approach). Bei den Testpersonen handelt es sich durchweg um Studenten der Universität Regensburg im Alter von 22 bis 26 Jahren. Alle Testpersonen wiesen bereits Erfahrung im Umgang mit Smartphones auf; somit konnte der Einfluss des „mobilen Endgeräts“ als Störvariable für den Usability-Test minimiert werden.

\subsection{Versuchsaufbau}

Die Evaluation wurde in einem ruhigen Raum mit wenig Ablenkung durchgeführt („Wohnzimmer-Setting"). Das gewählte Umfeld erleichterte zudem die Datenerhebung in Form von Screenvideos und think aloud-Protokollen, da Störquellen weitestgehend vermieden werden konnten. Eine Sitzung dauerte jeweils ca. eine Stunde, wobei jeder der Probanden alle drei Browser randomisiert mit sechs Testszenarien zu acht Hauptfunktionen mobiler Browser testete. Als Testgeräte kamen dabei ein iPhone 4 (Betriebssystem: iOS 6) von Apple für die Browser Safari und Opera Mini zum Einsatz, während für Androids Stock Browser ein HTC Sensation (Betriebssystem: JellyBean, Ver. 4.1.2) verwendet wurde.

\subsection{Testablauf}

$\mathrm{Zu}$ Beginn jeder Testsitzung wurden den Studienteilnehmern die wesentlichen Ziele der Evaluation kurz erläutert (briefing). Dabei wurden neben der allgemeinen Vorgehensweise auch die Methoden der Datenerhebung erklärt: Einerseits erfolgte die Datenerfassung durch die Aufzeichnung des Bildschirms sowie der Äußerungen während der Evaluation. Andererseits wurden vom Testleiter Schlüsselereignisse und Aussagen der Testpersonen in think aloud-Protokollen schriftlich dokumentiert. Danach füllten die Studienteilnehmern eine Einverständniserklärung zur Nutzung der Daten und einen Fragebogen zur Erhebung demografischer Daten und Informationen zur Smartphone- und Interneterfahrung aus. Anschließend wurden die drei Browser in randomisierter Reihenfolge getestet. Dazu mussten die 
Testpersonen mit jedem Web-Browser die gleichen sechs Testszenarien absolvieren. Im Anschluss an den Test eines Browsers wurde jeweils eine Teilmenge der Fragen des UEQ beantwortet. Nachdem alle drei Browser getestet wurden erfolgte ein debriefing der Teilnehmer, in dem mögliche Fragen zum Testablauf oder zu speziellen Handlungsschritten gemeinsam diskutiert wurden. Somit wurde sichergestellt, dass Unklarheiten für die Auswertung aufgelöst wurden.

\subsection{Testmaterialien}

Der zu Beginn jeder Testsitzung gestellte Fragebogen umfasste Fragen zu demografischen Daten wie etwa Alter, Geschlecht, Beruf und Schulbildung. Ferner wurden Informationen zu Vorkenntnissen der Nutzer im Umgang mit Internet und Web-Browsern sowie die allgemeine Erfahrung mit Smartphones erfasst. Zudem erhielten alle Studienteilnehmern sechs Aufgaben in gedruckter Form, die sicherstellen, dass die zuvor ermittelten Hauptfunktionen, die alle drei Browser gemeinsam haben, während der Evaluation von den Nutzern exploriert werden.

Beispiel: Aufgabe (1) Sie sind auf der Suche nach neuen Kleidungsstücken und möchten dazu auf Zalando etwas Passendes finden. Besuchen Sie www.zalando.de und wählen ein beliebiges Kleidungsstück für die nähere Betrachtung aus. Da Ihnen das Kleidungsstück gefällt, möchten Sie die Detailseite als Lesezeichen speichern.

Im Anschluss an den Test jedes Browsers erhielten die Probanden einen Fragebogen zur Erfassung der Usability der Testobjekte. Dazu wurde aus dem UEQ die Dimension der Benutzungsqualität isoliert. Die beiden anderen Dimensionen (Attraktivität und Designqualität) sind für die Erhebung der Usability nicht relevant, und wurden deshalb zur Minimierung der Belastung für die Testpersonen aus dem Fragebogen entfernt.

\section{Diskussion der Ergebnisse}

Bei der Auswertung der Evaluationsergebnisse wurden die Daten des UEQ mit den Usability-Problemen aus den think aloud-Protokollen abgeglichen. Zur Vorbereitung wurden die Probleme nach Schweregrad und Auftretenswahrscheinlichkeit sortiert (vgl. Rubin \& Chisnell, 2008). Die Mittelwerte für die drei Skalen „Durchschaubarkeit“, „Effizienz“ und „Steuerbarkeit" der Dimension der Benutzungsqualität des UEQ liegen zwischen +3 und -3 . Dabei entsprechen Werte zwischen +1 und -1 einer neutralen Beurteilung. Niedrigere Werte als -1 entsprechen einer negativen, und Werte höher als +1 einer positiven Bewertung. Da Extremwerte bei Likert-Skalen vermieden werden sollten, ergibt sich ein Wertebereich zwischen +2 und -2 . Werte an der oberen oder unteren Grenze dieses Felds können als signifikant positiv bzw. negativ interpretiert werden (Rauschenberger, Schrepp, Perez-Cota, Olschner \& Thomaschewski, 2013). Tabelle 3 fasst die quantitativen Ergebnisse zusammen: 


\begin{tabular}{|c|c|c|c|c|c|c|}
\hline Funktion & Browser & $\begin{array}{c}\text { Durchschau- } \\
\text { barkeit }\end{array}$ & Effizienz & $\begin{array}{l}\text { Steuer- } \\
\text { barkeit }\end{array}$ & $\begin{array}{l}\text { Usability- } \\
\text { Probleme }\end{array}$ & $\begin{array}{l}\text { Durchschnitt. } \\
\text { Severity Rank }\end{array}$ \\
\hline \multirow{3}{*}{$\begin{array}{c}\text { Aufrufen einer } \\
\text { URL }\end{array}$} & Android & 2,125 & 1,500 & 2,083 & 1 & 3 \\
\hline & Safari & 1,917 & 0,792 & 1,792 & 1 & 3 \\
\hline & Opera & 2,000 & 1,333 & 1,875 & 2 & 3 \\
\hline \multirow{3}{*}{$\begin{array}{l}\text { Suche in einer } \\
\text { Suchmaschine }\end{array}$} & Android & 2,542 & 2,125 & 2,292 & 1 & 3 \\
\hline & Safari & 1,625 & 1,458 & 1,292 & 1 & 3 \\
\hline & Opera & 2,000 & 1,708 & 1,583 & 1 & 4 \\
\hline \multirow{3}{*}{$\begin{array}{l}\text { Vorwärts- / } \\
\text { Rückwärts- } \\
\text { navigation }\end{array}$} & Android & 1,750 & 1,542 & 1,708 & 1 & 3 \\
\hline & Safari & 1,958 & 1,708 & 1,958 & 1 & 3 \\
\hline & Opera & 1,417 & 1,500 & 1,167 & 3 & 3 \\
\hline \multirow{3}{*}{$\begin{array}{c}\text { Surfen mit } \\
\text { Tabs / Fenstern }\end{array}$} & Android & 1,917 & 1,375 & 1,708 & 1 & 6 \\
\hline & Safari & 1,917 & 1,000 & 1,833 & 0 & 0 \\
\hline & Opera & 1,458 & 1,333 & 1,667 & 0 & 0 \\
\hline \multirow{3}{*}{$\begin{array}{l}\text { Verwalten von } \\
\text { Lesezeichen }\end{array}$} & Android & 1,875 & 1,792 & 1,958 & 2 & 3 \\
\hline & Safari & $-0,208$ & 0,000 & $-0,167$ & 5 & 3,8 \\
\hline & Opera & 0,917 & 0,958 & 0,750 & 4 & 3,5 \\
\hline \multirow{3}{*}{$\begin{array}{l}\text { Anwendung des } \\
\text { Surfverlaufs }\end{array}$} & Android & 1,792 & 1,083 & 2,000 & 2 & 5 \\
\hline & Safari & 1,167 & 0,958 & 0,792 & 0 & 0 \\
\hline & Opera & 1,875 & 1,125 & 1,625 & 0 & 0 \\
\hline \multirow{3}{*}{$\begin{array}{l}\text { Speichern für } \\
\text { Offline-Zugriff }\end{array}$} & Android & 1,625 & 1,542 & 1,708 & 0 & 0 \\
\hline & Safari & 0,167 & 0,667 & 0,417 & 3 & 5 \\
\hline & Opera & 0,542 & 0,583 & 0,667 & 3 & 5 \\
\hline \multirow{3}{*}{$\begin{array}{l}\text { Durchsuchen } \\
\text { einer Seite }\end{array}$} & Android & 1,958 & 1,708 & 1,958 & 0 & 0 \\
\hline & Safari & $-1,000$ & 0,750 & $-0,417$ & 4 & 6 \\
\hline & Opera & 1,458 & 1,625 & 1,875 & 1 & 3 \\
\hline
\end{tabular}

Tabelle 3: Mittelwerte des UEQ.

Im Folgenden werden die wesentlichen Ergebnisse für jede der Browser-Hauptfunktionen diskutiert, und als Fazit für die Entwickler künftiger Browser zusammengefasst.

\section{Funktion: Aufrufen einer URL}

Im ersten der sechs gestellten Tasks bestand die Aufgabe darin, eine vorgegebene URL aufzurufen. In allen drei Browsern findet sich dazu eine Eingabeleiste am oberen Rand der Anwendung. Am besten wird bei dieser Funktion Googles Android Stock Browser bewertet, vermutlich da durch den Verzicht auf eine separate Suchleiste (statt dessen: Omnibox) eine bessere Übersichtlichkeit entsteht. Einige Testpersonen bemängelten, dass bei Safari während des Ladens einer Seite keine weitere Interaktion möglich ist.

Fazit: Kombination von URL-Adressleiste und Suchleiste wird am besten bewertet.

\section{Funktion: Suche in einer Suchmaschine}

Zur Untersuchung der Integration von Suchmaschinen in den drei Browsern sollten die Probanden über die Suchmaschine Google einen Artikel in der Wikipedia aufrufen. Auch bei 
dieser Funktion wird der Android Stock Browser durch die einzelne, multifunktionale Eingabeleiste (Omnibox) besser als seine Konkurrenten bewertet. Die beiden Browser Safari und Opera nutzen für die Formulierung einer Anfrage an die eingestellte Suchmaschine ein eigenes Eingabefeld, das allerdings von einigen Probanden übersehen wird. Alle drei Browser werden für diese Hauptaufgabe eines mobilen Browsers durchweg positiv beurteilt, was sich auch in der geringen Anzahl der Usability-Probleme niederschlägt.

Fazit: Kombination von URL-Adressleiste und Suchleiste wird am besten bewertet.

\section{Funktion: Vorwärts- / Rückwärtsnavigation}

In der Funktion zur Vorwärts- und Rückwärtsnavigation innerhalb des Browsers liegen die Bewertungen für die drei Browser in allen Skalen der Benutzungsqualität des UEQ im positiven Bereich. In den Browsern Safari und Opera ist die Funktion über zwei Pfeile am unteren Rand des Anwendungsbereichs realisiert, bei Android Stock Browser erfolgt die Rückwärtsnavigation über den standardmäßigen Hardware-Button und die Vorwärtsnavigation über das Optionsmenü.

Fazit: Im Falle des Android-Browsers liegt es nahe, die auf dieser Plattform etablierte Funktion (Hardware-Button für ,ein Schritt zurück“) für die Rückwärtsnavigation im Browser zu verwenden; ansonsten sollten sich mobile Browser an Navigationselementen orientieren, die für den Desktop-Bereich bereits etabliert sind.

\section{Funktion: Surfen mit Tabs / Fenstern}

In zwei der sechs Tasks wurde die Funktion zum Surfen mit Fenstern überprüft. Bei den Browsern Safari und Opera kam es dabei zu keinerlei Usability-Problemen. Einige Nutzer merkten zur Schaltfläche des Android Stock Browser zur Auslösung des Features an, dass diese nicht die Funktion zum Wechseln von Fenstern suggeriert. Des Weiteren verbirgt sich hinter der genannten Schaltfläche nicht nur die benötigte Funktion, sondern darüber hinaus auch noch Buttons zum Browserverlauf und den Lesezeichen. Da im Android Stock Browser viele Funktionen in das Optionsmenü verlagert wurden, vermuteten einige Nutzer die Schaltfläche zur Fensterverwaltung zunächst ebenfalls darin.

Fazit: Safari und Opera verwenden ein weitgehend konventionalisiertes Icon für das Öffnen von Tabs, das sich zudem an der gleichen Position, am unteren Bildschirmrand befindet. Das Surfen mit mehreren Tabs / Fenstern ist eine essenzielle Funktion mobiler Browser und sollte direkt mit einem Klick erreichbar sein und nicht mit anderen Funktionen (Bookmarks, Verlauf, etc.) in einem Menü vermischt werden.

\section{Funktion: Verwalten von Lesezeichen}

Bei der Lesezeichenverwaltung wurde der Android Stock Browser durchweg besser bewertet als die beiden anderen Browser. Dies ist vor allem durch die spezifischen Usability-Probleme von Safari und Opera zu erklären. Bei Safari vermissten manche Probanden die Möglichkeit zum Öffnen von Kontextmenüs mit einem langen Klick. Weitere Probleme bei Safari hängen mit der Gestaltung der Lesezeichenliste und dem häufig nicht gefundenen Button zum Hin- 
zufügen eines Lesezeichens über das „Teilen“-Menü zusammen. Bei Operas Browser trat mehrfach das Problem auf, dass die Nutzer den Button zum Hinzufügen von Lesezeichen in Form eines Plus-Icons, mit dem Favicon der Webseite verwechselten. Des Weiteren wurde mangelndes Feedback und die Sortierung der Lesezeichenliste bemängelt.

Fazit: Während bei Opera und Safari das Erstellen neuer Lesezeichen über die jeweiligen Icons nicht intuitiv ist, kann die Funktion beim Android-Browser direkt über den HardwareButton „Kontext-Menü“ erreicht werden, wo sie als gut verständliche Wortmarke („Lesezeichen hinzufügen") aufgeführt ist.

\section{Funktion: Anwendung des Surfverlaufs}

In einem weiteren Task sollte eine bereits aufgerufene Seite über den Surfverlauf der Browser erneut besucht werden. Bei den beiden Browsern Opera und Safari traten dabei keinerlei Schwierigkeiten auf. Trotz der positiven Beurteilungen für den Android Stock Browser wurden bei dieser Funktion einige Usability-Probleme entdeckt: Durch die Auslagerung zentraler Funktionen in das Android-spezifische Optionsmenü vermuteten einige Nutzer auch den Surfverlauf dort. Durch die Platzierung dieser Funktion im gleichen Menü, in dem auch Fenster und Lesezeichen verwaltet werden können, und das ungünstig gewählte Icon zu diesem Menü, traten bei einigen Probanden ebenfalls Probleme auf.

Fazit: Bei Safari und Opera kam es zu keinerlei Problemen. Bei Opera ist die Funktion des Surfverlaufs im Menü des Browsers untergebracht. Safari platziert den Verlauf im Lesezeichenmenü oberhalb der eigentlichen Lesezeichen. Ein Problem im Android Stock Browser ist die stark erschwerte Erreichbarkeit des Surfverlaufs.

\section{Funktion: Speichern einer Seite für den Offline-Zugriff}

Die Möglichkeit zur Speicherung von Webseiten für den Offline-Zugriff ist hauptsächlich bei mobilen Browsern zu finden und ermöglicht das Lesen von zuvor gespeicherten Seiten auch in Situationen, in denen kein Internetzugang möglich ist. Bei dieser Funktion wird der Android Stock Browser am besten bewertet. In Safari wird die Funktion als Leseliste bezeichnet, was bei einigen Nutzern zu Verwirrung führte. Das bereits bei der Funktion zum Hinzufügen von Lesezeichen erwähnte Icon in Form eines Plus-Zeichens kommt bei Operas Browser auch bei der Speicherung von Seiten für den Offline-Zugriff zum Einsatz, und wird hier ebenfalls mit dem Favicon der Seite verwechselt. Auch bei dieser Funktion wurde von einigen Nutzern die Sortierung der bereits gespeicherten Seiten bemängelt. Ein weiteres Manko bei Opera stellt die Schaltfläche für den Offline-Zugriff im Menü dar. Das Icon in Form einer Kamera und das Label führte bei einigen Nutzern zu Verwirrung.

Fazit: Der Android Stock Browser platziert die Funktion zum Speichern von Seiten im bereits erwähnten, betriebssystem-spezifischen Kontextmenü. Ein Problem stellt - wie bei der Funktion des Surfverlaufs - die schwere Erreichbarkeit der gespeicherten Seiten dar. Allerdings führte dies bei den Nutzern zu keinen großen Schwierigkeiten, da die Navigationsschritte bereits aus vorangehenden Tasks bekannt waren; dies lässt wiederum auf ein leicht zu erlernendes Interaktionskonzept schließen. 


\section{Funktion: Durchsuchen einer Webseite}

Auch bei der Funktion zum Durchsuchen von Webseiten treten beim Android Stock Browser keinerlei Probleme auf. Bei Opera wurde die ungenügende Hervorhebung, falls die Suche keine Treffer ergibt, bemängelt. In Safari traten bei der Evaluation dieser Funktion erhebliche Mängel auf: Fünf der sechs Testpersonen mussten die Aufgabe frühzeitig abbrechen, da sie die Funktion im Browser nicht fanden. Lediglich ein Proband erkannte die Suchleiste am rechten, oberen Rand des Browsers, die gewöhnlich zur Suche in Suchmaschinen verwendet wird, als Möglichkeit zur Suche auf der Seite. Allerdings führte das fehlende Feedback, wenn keine Treffer gefunden wurden, zu dem Problem, dass durch das Drücken der Bestätigen-Taste in der Tastatur die Google-Suche ausgelöst wurde.

Fazit: Suchfenster im rechten, oberen Bildschirmbereich (direkt neben der URL-Eingabe) werden als Möglichkeit der Web-Suche erkannt (gelerntes Verhalten aus dem DesktopBereich). Aus diesem Grund sollte die eher selten verwendete Funktion der seiteninternen Suche nicht an dieser Stelle platziert werden, sondern in ein Kontextmenü ausgelagert werden.

\section{Zusammenfassung}

Dieser Beitrag beschreibt eine vergleichende Usability-Evaluation von drei häufig genutzten Web-Browsern für mobile Endgeräte. Dabei wurden zunächst wesentliche Funktionen mobiler Browser mithilfe eines explorativen Walkthroughs ermittelt. Diese Funktionen wurden dann in einer Benutzerstudie in Form spezifischer Tasks evaluiert. Es zeigt sich, dass typische Funktionen teilweise sehr unterschiedlich von den unterschiedlichen Browsern umgesetzt werden, und die verschiedenen Browser dementsprechend unterschiedliche UsabilityProbleme aufweisen. Die Ergebnisse mögen als Anhaltspunkt für Browser-Entwickler dienen, um die erkannten Schwächen der jeweiligen Browser zu optimieren, oder Anwender bei der Auswahl eines benutzerfreundlichen, mobilen Browsers unterstützen. Zudem können die identifizierten Hauptfunktionen mobiler Browser als Grundlage für weitere Evaluationsstudien (andere Browser / mehr Testpersonen / etc.) herangezogen werden, und mit kleineren Anpassungen auch auf den Desktop-Bereich übertragen werden.

\section{Literaturverzeichnis}

Accenture. (2011). Mobile Web Watch 2011. < http://www.accenture.com/SiteCollectionDocuments/ Local_Germany/PDF/Accenture-Studie-Mobile-Web-Watch-2011.pdf $>$, letzter Zugriff am 19.3.2014

Accenture. (2012). Mobile Web Watch 2012.<http://www.accenture.com/SiteCollectionDocuments/ $\mathrm{PDF} /$ Accenture-Study-Mobile-Web-Watch-Germany-Austria-Switzerland-EN.pdf $>$, letzter Zugriff am 19.3.2014 
Gardner, B. S. (2011). Responsive Web Design: Enriching the User Experience. In: Sigma (Noblis Technical Journal) 11(1), 13-19.

Google, \& Nielsen. (2013). Mobile Search Moments Study: Understanding how mobile drives conversions. <http://www.google.com/think/research-studies/creating-moments-that-matter.html>, letzter Zugriff am 19.3.2014

Laugwitz, B., Schrepp, M., \& Held, T. (2006). Konstruktion eines Fragebogens zur Messung der User Experience von Softwareprodukten. In: Heinicke, A. (Hg.) Mensch und Computer im Strukturwandel. Proc. Mensch \& Computer 2006, Gelsenkirchen, September 2006, München / Wien: Oldenbourg Verlag, 125-134.

Marcotte, E. (2010). Responsive Web Design. A List Apart. <http://alistapart.com/article/responsiveweb-design>, letzter Zugriff am 19.3.2014

Nielsen, J. (1998). Cost of User Testing a Website. <http://www.nngroup.com/articles/cost-of-usertesting-a-website/>, letzter Zugriff am 19.3.2014

Rauschenberger, M., Schrepp, M., Perez-Cota, M., Olschner, S., \& Thomaschewski, J. (2013). Efficient Measurement of the User Experience of Interactive Products. How to use the User Experience Questionnaire (UEQ).Example: Spanish Language Version. International Journal of Interactive Multimedia and Artificial Intelligence, 2(1), 39-45.

Roto, V., \& Kaikkonen, A. (2003). Perception of Narrow Web Pages on a Mobile Phone. In Proc. 19th International Symposium on Human Factors in Telecommunications 2003 (HFT 2003), Berlin, December 2003. Boston: Information Gatekeepers, 187-194.

Rubin, J., \& Chisnell, D. (2008). Handbook of usability testing (2nd ed.). Indianapolis: Wiley.

Shrestha, S. (2007). Mobile web browsing. In Chong, P. H. J.; Cheok, A. D. (Hrsg.) Proc. $4^{\text {th }}$ International Conference on Mobile Technology, Applications, and Systems (Mobility 2007), Singapur, September 2007. New York: ACM Press, 187-194.

Voigts, R., Christmann, S., \& Hagenhoff, S. (2011). Mobile Web Browsers (Working Paper No. 01). $<$ http://www2.as.wiwi.uni-goettingen.de/getfile?DateiID=713>, letzter Zugriff am 19.3.2014

Wroblewski, L. (2011). Mobile First. New York: A Book Apart.

\section{Kontaktinformationen}

Manuel Burghardt

Lehrstuhl für Medieninformatik

Universität Regensburg

93040 Regensburg

E-Mail: manuel.burghardt@ur.de

Webseite: www.mi.ur.de 